\title{
A scoping review of palliative care outcome measures in interstitial lung disease
}

\author{
Rebecca A. Gersten $\mathbb{C}^{1,4}$, Amanda C. Moale $\mathbb{C}^{2,4}$, Bhavna Seth ${ }^{1}$, Judith B. Vick ${ }^{2}$, Hannah Brown ${ }^{3}$, \\ Michelle N. Eakin ${ }^{1}$, Stephen C. Mathai ${ }^{1}$ and Sonye K. Danoff ${ }^{1}$
}

${ }^{1}$ Division of Pulmonary and Critical Care Medicine, Johns Hopkins University, Baltimore, MD, USA. ${ }^{2}$ Dept of Medicine, Johns Hopkins Hospital, Baltimore, MD, USA. ${ }^{3}$ Dept of Medicine, Johns Hopkins Bayview Medical Center, Baltimore, MD, USA. ${ }^{4}$ Joint first authors.

Corresponding author: Rebecca A. Gersten (rgerste4@jhu.edu)

Shareable abstract (@ERSpublications)

Palliative care is a key component of quality care for patients with interstitial lung disease (ILD). This study delineates outcome measures used to assess palliative care in ILD and highlights the current heterogeneity, which hinders comparison between studies. https://bit.ly/3p8CbXQ

Cite this article as: Gersten RA, Moale AC, Seth B, et al. A scoping review of palliative care outcome measures in interstitial lung disease. Eur Respir Rev 2021; 30: 210080 [DOI: 10.1183/16000617.00802021].

Copyright @The authors 2021

This version is distributed under the terms of the Creative Commons Attribution NonCommercial Licence 4.0. For commercial reproduction rights and permissions contact permissions@ersnet.org

This article has supplementary material available from err.ersjournals.com

Received: 19 March 2021 Accepted: 26 May 2021

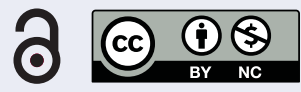

\section{Abstract}

Interstitial lung disease (ILD) confers a high mortality and symptom burden, substantially impacting quality of life. Studies evaluating palliative care in ILD are rapidly expanding. Uniform outcome measures are crucial to assessing the impact of palliative care in ILD. This scoping review evaluates existing outcome measures in general health-related quality of life (HRQoL), physical health, mental health, social health and advance care planning (ACP) domains in patients with ILD. Articles in English with quantitative assessment of at least one measure of general HRQoL, physical health, mental health, social health or ACP in patients with ILD were included. Searches across three databases yielded 3488 nonduplicate articles. 23 met eligibility criteria and included three randomised controlled trials (RCTs) or secondary analysis of an RCT (13\%), three cross-sectional studies or secondary analysis of cross-sectional study (13\%), one prospective study (4\%) and 16 retrospective studies (70\%). Among eligible articles, 25 distinct instruments were identified. Six studies assessed general HRQoL (26\%), 16 assessed physical health (70\%), 11 assessed mental health (48\%), six assessed social health (26\%) and 16 assessed ACP (70\%). The ability to compare results across studies remains challenging given the heterogeneity in outcome measures. Future work is needed to develop core palliative care outcome measures in ILD.

\section{Introduction}

\section{Rationale}

Interstitial lung disease (ILD) is a group of lung diseases with distinct aetiologies but common pathways of inflammation and fibrosis. The prevalence of ILD is 97.9 per 100000 persons with an incidence of 19.4 per 100000 person-years based on a recent study from France [1]. ILD affects individuals across a range of ages, races and sexes. Treatment is limited and, even with advanced therapy, the median survival ranges from 2-3 years in idiopathic pulmonary fibrosis [2] to 7 years in both chronic hypersensitivity pneumonitis [3] and rheumatoid arthritis-associated ILD [4]. For patients who require supplemental oxygen, the median survival decreases to 8.4 months [5]. In addition to the high mortality rate, ILD is associated with a profound symptom burden, including intractable cough, fatigue and immobilising dyspnoea [6]. In fact, ILD patients have more severe dyspnoea at the end of life than terminal lung cancer patients [7]. In addition to physical suffering, ILD patients may experience psychological distress, social impairment and difficult decisions regarding advance care planning (ACP) [8].

Palliative care aims to improve quality of life (QoL) through identification, assessment and treatment of physical, psychological, emotional and spiritual distress, while determining patients' values, life goals and preferences around dying [9]. The American Thoracic Society recommends that palliative care be available 
and tailored to patients at all stages of illness and has prioritised research funding that advances high-quality palliative care and improves clinically relevant outcomes [9]. Clinically relevant palliative care outcomes assess patients' symptoms and needs, monitor changes in health status or QoL, facilitate communication, aid in clinical decision-making and evaluate the effect of interventions or care [10]. Heterogeneity of outcome measures limits the ability of comparing the efficacy of interventions across studies. Thus, standardisation of a core outcome set is needed [10]. An essential first step in this process is to identify key domains and summarise existing palliative care outcome measures in patients with ILD via a scoping review.

\section{Objectives}

This scoping review seeks to delineate the palliative care outcome measures used in palliative care-related observational studies and clinical trials of patients with ILD and to classify them into five distinct domains. We use the validated patient-reported outcomes measurement information system (PROMIS) domains: physical health, mental health and social health. The PROMIS domains were used to provide a consistent, recognised methodology $[11,12]$. In this review, it was recognised that there are data that fall into two important domains not easily categorised under the PROMIS structure. Thus, we propose two additional domains: general health-related quality of life (HRQoL) and ACP. This scoping review may serve as a reference for future researchers selecting palliative care outcome measures in the ILD population.

\section{Methods}

Identification of eligible studies

We conducted a search of palliative care interventions in patients with ILD. Our initial search was conducted on 19 August 2019 and included PubMed, Embase and the Cochrane Library. Major search terms included: "palliative care", "hospice and palliative care” and "palliation”, in addition to general and more specific search terms for the major types of advanced lung disease, including ILD. We limited searches to citations in English (supplementary material). An updated search was conducted on 31 October 2020 and we identified two additional studies that met inclusion criteria.

\section{Study selection}

Exclusion criteria included articles that addressed a population aged $<18$ years, did not address palliative care, only included qualitative data, described surgical procedures, case studies of less than 10 cases and editorials or other descriptive nonclinical articles. We screened article titles and abstracts, reviewed full texts of articles, and finally performed data abstraction. Reviewers were all educated on inclusion and exclusion criteria, and we conducted regular team meetings to identify any concerns. Two independent reviewers screened all citations. Discrepancies were resolved through a third reviewer or consensus agreement. Abstract and full-text screening was managed using Covidence Software (Veritas Health Innovation, Melbourne, Australia). Reviewers (A.C. Moale, B. Seth, H. Brown, J.B. Vick and R.A. Gersten) initially screened titles and abstracts of each retrieved citation and subsequently reviewed eligible full text articles for inclusion. Only studies that addressed at least one palliative care outcome in patients with ILD with quantitative data were included. Studies that combined both qualitative and quantitative data were included, but only quantitative data were abstracted.

\section{Data abstraction}

Data abstraction was performed by A.C. Moale and R.A. Gersten between February and October 2020. The following data were collected from each of the eligible articles: author, year, study location, study design, aims of study, outcomes measured and instruments used to assess each outcome (table 1). Validated tools, custom-made instruments/questionnaires and numerical scales were all included under instruments. The term "retrospective data" includes outcomes assessed via retrospective chart review, the medical record and/or care provider discussions.

Outcome domains were categorised according to the physical, mental and social health PROMIS Adult Self-Reported Health domains with the addition of general HRQoL and ACP, as fourth and fifth domains, respectively (table 2). HRQoL was included as a separate domain for the measurement of general HRQoL, rather than a specific physical, mental or social health dimension of QoL (figure 1). Social health includes social function, defined by the ability to participate in and satisfaction with social roles and social relationships, such as social support rather than isolation [34]. Table 2 lists the outcomes measured under each domain. The instrument(s) used to measure each outcome was identified (tables 1 and 3) according to which outcome and domain each article used the instrument to assess. Instruments were included under multiple domains if the authors used the instrument to assess outcomes in multiple domains. 
TABLE 1 Description of all 23 included studies

\begin{tabular}{|c|c|c|c|c|c|}
\hline $\begin{array}{l}\text { First author } \\
\text { [ref.], study } \\
\text { location }\end{array}$ & $\begin{array}{l}\text { Intervention } \\
\text { type }\end{array}$ & $\begin{array}{l}\text { Study population } \\
\text { sample size }\end{array}$ & Aims of study & Outcomes measured & $\begin{array}{l}\text { Method of data } \\
\text { collection used to } \\
\text { assess outcomes }\end{array}$ \\
\hline $\begin{array}{l}\text { АнмаDI [5], } \\
\text { Sweden }\end{array}$ & Retrospective & $\begin{array}{l}\text { Patients dying with } \\
\text { oxygen- dependent } \\
\text { ILD versus lung } \\
\text { cancer, } 285 \text { patients } \\
\text { with ILD versus } 10 \\
822 \text { patients with } \\
\text { lung cancer }\end{array}$ & $\begin{array}{l}\text { To compare prevalence of } \\
\text { symptoms and treatments } \\
\text { between two groups }\end{array}$ & $\begin{array}{c}\text { Physical: } \\
\text { Dyspnoea } \\
\text { GI symptoms (nausea) } \\
\text { Pain } \\
\text { Mental: } \\
\text { Anxiety } \\
\text { Cognitive function } \\
\text { (confusion) } \\
\text { ACP: } \\
\text { Expected versus unexpected } \\
\text { death } \\
\text { GOC discussions } \\
\text { LOD } \\
\text { Specialist PC (consultation) }\end{array}$ & SRPC EOL Questionnaire \\
\hline $\begin{array}{l}\text { AKHTAR [13], } \\
\text { UK }\end{array}$ & Prospective & $\begin{array}{l}\text { IPF patients who } \\
\text { attended ILD clinic } \\
\quad(n=118)\end{array}$ & $\begin{array}{l}\text { Determine prevalence of } \\
\text { depressive symptoms }\end{array}$ & $\begin{array}{c}\text { Mental: } \\
\text { Depression }\end{array}$ & Wakefield-SADI \\
\hline $\begin{array}{l}\text { ARCHIBALD [14], } \\
\text { Canada }\end{array}$ & Retrospective & $\begin{array}{l}\text { ILD patients seen in } \\
\text { MDC clinic }(n=92)\end{array}$ & $\begin{array}{c}\text { Explore effects of PC bundle } \\
\text { on LOD }\end{array}$ & $\begin{array}{c}\text { Physical: } \\
\text { Dyspnoea } \\
\text { Other (cough) } \\
\text { Social: } \\
\text { Social isolation } \\
\text { ACP: } \\
\text { Specialist PC } \\
\text { ACP activities } \\
\text { LOD }\end{array}$ & $\begin{array}{c}\text { mMRC } \\
\text { SDI } \\
\text { Retrospective data }\end{array}$ \\
\hline $\begin{array}{l}\text { BaJWAH [15], } \\
\text { UK }\end{array}$ & Retrospective & $\begin{array}{l}\text { PIF-ILD in two } \\
\text { London ILD centres } \\
\quad(n=45)\end{array}$ & $\begin{array}{l}\text { To assess PC needs, use of PC } \\
\text { treatments, and whether EOL } \\
\text { preferences were documented } \\
\text { and achieved }\end{array}$ & $\begin{array}{c}\text { Physical: } \\
\text { Dyspnoea } \\
\text { Fatigue } \\
\text { GI symptoms (dyspepsia, } \\
\text { dysphagia and diarrhoea) } \\
\text { Pain } \\
\text { Sleep disturbance } \\
\text { Other (cough, weight loss, } \\
\text { chest pain, polyuria/ } \\
\text { polydipsia and headache) } \\
\text { Mental: } \\
\text { Depression/anxiety } \\
\text { ACP: } \\
\text { LOC (preferred) } \\
\text { LOD (preferred and actual) } \\
\text { Specialist PC }\end{array}$ & Retrospective data \\
\hline $\begin{array}{l}\text { BaJWAH [16], } \\
\text { UK }\end{array}$ & RCT & $\begin{array}{l}53 \text { patients with } \\
\text { advanced fibrotic ILD } \\
\text { and their carers ( } 26 \\
\text { patients randomised } \\
\text { to intervention in } \\
4 \text { weeks, } 27 \text { later } \\
\text { randomised to } \\
\text { intervention) }\end{array}$ & $\begin{array}{l}\text { Impact of case conference } \\
\text { intervention delivered in } \\
\text { home on PC concerns of } \\
\text { patients and their carers }\end{array}$ & $\begin{array}{c}\text { HRQoL: } \\
\text { Patient QoL } \\
\text { Physical: } \\
\text { Dyspnoea } \\
\text { Fatigue } \\
\text { Physical function } \\
\text { Sleep disturbance } \\
\text { Other (cough) } \\
\text { Mental: } \\
\text { Anxiety } \\
\text { Depression } \\
\text { ACP: } \\
\text { LOC (preferred) } \\
\text { LOD (preferred) }\end{array}$ & $\begin{array}{c}\text { KB-ILD } \\
\text { SGRQ } \\
\text { D12 Scale } \\
\text { MRC } \\
\text { POS } \\
\\
\text { HADS }\end{array}$ \\
\hline
\end{tabular}




\section{TABLE 1 Continued}

\begin{tabular}{|c|c|c|c|c|c|}
\hline $\begin{array}{l}\text { First author } \\
\text { [ref.], study } \\
\text { location }\end{array}$ & $\begin{array}{l}\text { Intervention } \\
\text { type }\end{array}$ & $\begin{array}{l}\text { Study population } \\
\text { sample size }\end{array}$ & Aims of study & Outcomes measured & $\begin{array}{l}\text { Method of data } \\
\text { collection used to } \\
\text { assess outcomes }\end{array}$ \\
\hline $\begin{array}{l}\text { BARRATT [17], } \\
\text { UK }\end{array}$ & Retrospective & $\begin{array}{l}72 \text { patients with } \\
\text { PIF-ILD ( } 46 \text { patients } \\
\text { under care of MDT } \\
\text { and } 26 \text { patients } \\
\text { pre-MDT care) }\end{array}$ & $\begin{array}{l}\text { To assess effectiveness of } \\
\text { multi-disciplinary team } \\
\text { meeting on patients' PC } \\
\text { needs }\end{array}$ & $\begin{array}{c}\text { ACP: } \\
\text { ACP activities (CPR } \\
\text { discussions) } \\
\text { Specialist PC }\end{array}$ & Retrospective data \\
\hline $\begin{array}{l}\text { BRown [18], } \\
\text { USA }\end{array}$ & $\begin{array}{l}\text { Secondary } \\
\text { analysis of } \\
\text { cluster RCT }\end{array}$ & $\begin{array}{l}829 \text { patients with } \\
\text { chronic lung disease } \\
\text { (COPD, ILD) and } \\
\text { metastatic cancer } \\
\text { (79 patients with ILD, } \\
592 \text { patients with } \\
\text { COPD, } 158 \text { patients } \\
\text { with metastatic } \\
\text { cancer) }\end{array}$ & $\begin{array}{l}\text { To explore differences in } \\
\text { receipt of PC among patients } \\
\text { with chronic lung disease who } \\
\text { die in ICUs compared with } \\
\text { cancer patients }\end{array}$ & $\begin{array}{c}\text { Physical: } \\
\text { Pain (pain assessment prior } \\
\text { to death) } \\
\text { ACP: } \\
\text { ACP activities (presence of } \\
\text { advance directive, presence } \\
\text { of DNR, avoidance of CPR) } \\
\text { GOC discussions (prognosis) } \\
\text { Mechanical support } \\
\text { (withdrawal of } \\
\text { life-sustaining measures) } \\
\text { Specialist PC }\end{array}$ & $\begin{array}{l}\text { Retrospective data } \\
\text { Retrospective data }\end{array}$ \\
\hline $\begin{array}{l}\text { HIGGINSON [19], } \\
\text { UK }\end{array}$ & RCT & $\begin{array}{l}105 \text { adults with } \\
\text { refractory } \\
\text { breathlessness and } \\
\text { advanced disease } \\
\text { (cancer, COPD, CHF, } \\
\text { ILD, MND) (53 } \\
\text { patients received } \\
\text { breathlessness } \\
\text { support service and } \\
52 \text { patients received } \\
\text { usual care) }\end{array}$ & $\begin{array}{l}\text { To assess effectiveness of } \\
\text { early PC with respiratory } \\
\text { services for patients with } \\
\text { advanced disease and } \\
\text { refractory breathlessness }\end{array}$ & $\begin{array}{c}\text { HRQoL: } \\
\text { (At } 6 \text { weeks) } \\
\text { Physical: } \\
\text { Dyspnoea (patient reported } \\
\text { breathlessness mastery) } \\
\text { Fatigue } \\
\text { Physical function } \\
\text { Mental: } \\
\text { Anxiety } \\
\text { Depression } \\
\text { Social: } \\
\text { Other (receipt of health, } \\
\text { voluntary or social care } \\
\text { services) }\end{array}$ & $\begin{array}{c}\text { CRQ } \\
\text { EQ-5D } \\
\text { EQ-5D-HRQOL-VAS } \\
\text { POS } \\
\text { CRQ } \\
\text { LCADL } \\
\text { NRS }\end{array}$ \\
\hline $\begin{array}{l}\text { KaLLURI [20], } \\
\text { Canada }\end{array}$ & Retrospective & $\begin{array}{c}32 \text { deceased IPF } \\
\text { patients before and } \\
\text { after MDC model ( } 22 \\
\text { patients in MDC care } \\
\text { and } 10 \text { in non-MDC } \\
\text { care) }\end{array}$ & $\begin{array}{c}\text { MDC model's association with } \\
\text { acute care utilisation in last } \\
\text { year of life and LOD }\end{array}$ & $\begin{array}{c}\text { ACP: } \\
\text { LOC (preferred) } \\
\text { LOD (preferred and actual) }\end{array}$ & Retrospective data \\
\hline $\begin{array}{l}\text { KovaUCHI [21], } \\
\text { Japan }\end{array}$ & Retrospective & $\begin{array}{l}84 \text { ILD patients with } \\
\text { DNI (54 patients on } \\
\text { HFNC and } 30 \\
\text { patients on NPPV) }\end{array}$ & $\begin{array}{l}\text { To assess efficacy and } \\
\text { tolerability of HFNC }\end{array}$ & $\begin{array}{c}\text { Physical: } \\
\text { Dyspnoea } \\
\text { Social: } \\
\text { Ability to participate in } \\
\text { social roles and activities } \\
\text { (communication ability with } \\
\text { family/ caregivers at the } \\
\text { EOL) }\end{array}$ & $\begin{array}{c}\text { STAS-J } \\
\text { Retrospective data }\end{array}$ \\
\hline LIANG [22], USA & Retrospective & $\begin{array}{c}\text { To describe } \\
\text { characteristics of IPF } \\
\text { patients referred to } \\
\text { specialty lung centre } \\
\quad(n=106)\end{array}$ & $\begin{array}{l}\text { To describe characteristics of } \\
\text { these patients who } \\
\text { experienced ICU admission, } \\
\text { including frequency and } \\
\text { timing of referral to PC }\end{array}$ & $\begin{array}{c}\text { ACP: } \\
\text { Specialist PC (\% referred to } \\
\text { PC before and during } \\
\text { admission) }\end{array}$ & Retrospective data \\
\hline $\begin{array}{l}\text { LINDELL [23], } \\
\text { USA }\end{array}$ & Retrospective & $\begin{array}{l}\text { To describe events } \\
\text { prior to death in IPF } \\
\text { patients managed at } \\
\text { specialty centre } \\
(n=404)\end{array}$ & $\begin{array}{l}\text { To describe time course of } \\
\text { events prior to death with a } \\
\text { focus on location of death } \\
\text { and timing of PC referral }\end{array}$ & $\begin{array}{c}\text { ACP: } \\
\text { LOD } \\
\text { Specialist PC (\% referred } \\
\text { and timing of referral) }\end{array}$ & Retrospective data \\
\hline
\end{tabular}




\section{TABLE 1 Continued}

\begin{tabular}{|c|c|c|c|c|c|}
\hline $\begin{array}{l}\text { First author } \\
\text { [ref.], study } \\
\text { location }\end{array}$ & $\begin{array}{l}\text { Intervention } \\
\text { type }\end{array}$ & $\begin{array}{l}\text { Study population } \\
\text { sample size }\end{array}$ & Aims of study & Outcomes measured & $\begin{array}{l}\text { Method of data } \\
\text { collection used to } \\
\text { assess outcomes }\end{array}$ \\
\hline $\begin{array}{l}\text { Matsunuma [7] } \\
\text { Japan }\end{array}$ & Retrospective & $\begin{array}{l}82 \text { patients with ILD } \\
\text { and lung cancer ( } 23 \\
\text { patients with ILD } \\
\text { and } 59 \text { patients with } \\
\text { lung cancer) }\end{array}$ & $\begin{array}{l}\text { To evaluate signs, symptoms, } \\
\text { and treatment before death }\end{array}$ & $\begin{array}{c}\text { Physical: } \\
\text { Dyspnoea } \\
\text { Fatigue } \\
\text { Pain } \\
\text { Sleep disturbance } \\
\text { (insomnia) } \\
\text { Other (cough, sputum, } \\
\text { anorexia) } \\
\text { Mental: } \\
\text { Cognitive function (loss of } \\
\text { consciousness and delirium) } \\
\text { ACP: } \\
\text { ACP activities (advance } \\
\text { directive) } \\
\text { Mechanical support } \\
\text { (intubation) }\end{array}$ & Retrospective data \\
\hline $\begin{array}{l}\text { RAJALA [24], } \\
\text { Finland }\end{array}$ & Retrospective & $\begin{array}{c}\text { Deceased IPF } \\
\text { patients in IPF } \\
\text { cohort study ( } n=59)\end{array}$ & $\begin{array}{c}\text { To describe treatment } \\
\text { practices, decision-making } \\
\text { and symptoms during } \\
\text { EOL care }\end{array}$ & $\begin{array}{c}\text { Physical: } \\
\text { Dyspnoea } \\
\text { GI symptoms (nausea and } \\
\text { constipation) } \\
\text { Pain } \\
\text { Other (cough) } \\
\text { Mental: } \\
\text { Anxiety/depression } \\
\text { Cognitive function (delirium) } \\
\text { ACP: } \\
\text { ACP activities (DNR orders) } \\
\text { GOC discussions (EOL } \\
\text { discussions) } \\
\text { LOD }\end{array}$ & $\begin{array}{l}\text { Retrospective data } \\
\text { Retrospective data }\end{array}$ \\
\hline $\begin{array}{l}\text { RAJALA [25], } \\
\text { Finland }\end{array}$ & Cross-sectional & IPF patients $(n=252)$ & $\begin{array}{l}\text { Primary aim: to investigate } \\
\text { HRQoL and symptoms } \\
\text { Secondary aim: to identify PC } \\
\text { needs by studying } \\
\text { relationship between mMRC, } \\
\text { HRQoL and symptoms }\end{array}$ & $\begin{array}{c}\text { HRQoL } \\
\text { physical: } \\
\text { Dyspnoea } \\
\text { Energy } \\
\text { Fatigue } \\
\text { GI symptoms (loss of } \\
\text { appetite, nausea and } \\
\text { constipation) } \\
\text { Pain } \\
\text { Physical function } \\
\text { Sleep disturbance } \\
\text { (insomnia) } \\
\text { Other (cough and dry } \\
\text { mouth) } \\
\text { Mental: } \\
\text { Anxiety } \\
\text { Depression } \\
\text { Social: } \\
\text { Ability to participate in } \\
\text { social roles and activities } \\
\text { (social functioning) }\end{array}$ & $\begin{array}{c}\text { RAND-36 } \\
\text { mMRC } \\
\text { mESAS } \\
\text { RAND-36 }\end{array}$ \\
\hline
\end{tabular}




\section{TABLE 1 Continued}

\begin{tabular}{|c|c|c|c|c|c|}
\hline $\begin{array}{l}\text { First author } \\
\text { [ref.], study } \\
\text { location }\end{array}$ & $\begin{array}{l}\text { Intervention } \\
\text { type }\end{array}$ & $\begin{array}{l}\text { Study population } \\
\text { sample size }\end{array}$ & Aims of study & Outcomes measured & $\begin{array}{l}\text { Method of data } \\
\text { collection used to } \\
\text { assess outcomes }\end{array}$ \\
\hline $\begin{array}{l}\text { Rajala [26], } \\
\text { Finland }\end{array}$ & Retrospective & $\begin{array}{l}\text { Deceased IPF } \\
\text { patients }(n=92)\end{array}$ & $\begin{array}{c}\text { To evaluate IPF patients' } \\
\text { symptoms and HRQoL in last } \\
2 \text { years of life }\end{array}$ & $\begin{array}{c}\text { HRQoL } \\
\text { physical: } \\
\text { Dyspnoea } \\
\text { Fatigue } \\
\text { GI symptoms (loss of } \\
\text { appetite) } \\
\text { Pain } \\
\text { Sleep disturbance } \\
\text { (insomnia) } \\
\text { Other (cough and dry } \\
\text { mouth) } \\
\text { Mental: } \\
\text { Anxiety } \\
\text { Depression } \\
\text { Social: } \\
\text { Social function } \\
\text { ACP: } \\
\text { LOD }\end{array}$ & $\begin{array}{c}\text { mESAS } \\
\text { NRS } \\
\text { RAND-36 } \\
\text { RAND-36 } \\
\text { Retrospective data }\end{array}$ \\
\hline ReILly [27], UK & $\begin{array}{c}\text { Secondary } \\
\text { analysis of } \\
\text { cross-sectional }\end{array}$ & $\begin{array}{c}88 \text { COPD, ILD and } \\
\text { cancer patients with } \\
\text { refractory } \\
\text { breathlessness ( } 53 \\
\text { patients with COPD, } \\
17 \text { patients with ILD, } \\
18 \text { patients with } \\
\text { cancer) }\end{array}$ & $\begin{array}{l}\text { To assess properties of LCADL } \\
\text { in patients with refractory } \\
\text { breathlessness due to } \\
\text { advanced disease }\end{array}$ & $\begin{array}{c}\text { HRQoL } \\
\text { Physical: } \\
\text { Dyspnoea } \\
\text { Other (other } \\
\text { symptoms) } \\
\text { Mental: } \\
\text { Anxiety } \\
\text { Depression } \\
\text { Social: } \\
\text { Ability to participate in } \\
\text { social roles and activities }\end{array}$ & $\begin{array}{l}\text { CRQ } \\
\text { LCADL } \\
\text { NRS } \\
\text { POS } \\
\text { POS-S } \\
\text { HADS } \\
\\
\text { LCADL }\end{array}$ \\
\hline $\begin{array}{l}\text { Rush [28], } \\
\text { Canada }\end{array}$ & Retrospective & $\begin{array}{l}\text { Mechanically } \\
\text { ventilated IPF } \\
\text { patients (3166) }\end{array}$ & $\begin{array}{l}\text { To examine the use of PC in } \\
\text { mechanically ventilated IPF } \\
\text { patients }\end{array}$ & $\begin{array}{c}\text { ACP: } \\
\text { ACP activities (DNR } \\
\text { status) } \\
\text { Mechanical support } \\
\text { (ventilation) } \\
\text { Specialist PC }\end{array}$ & Retrospective data \\
\hline $\begin{array}{l}\text { SmalLwood [29], } \\
\text { Australia }\end{array}$ & Retrospective & $\begin{array}{l}\text { Patients with fibrotic } \\
\qquad \operatorname{ILD}(n=67)\end{array}$ & $\begin{array}{l}\text { To examine care at terminal } \\
\text { hospital admission and the } \\
\text { past } 2 \text { years of life }\end{array}$ & $\begin{array}{c}\text { Physical: } \\
\text { Dyspnoea } \\
\text { ACP: } \\
\text { ACP activities (including } \\
\text { code status) } \\
\text { Mechanical support } \\
\text { Specialist PC }\end{array}$ & $\begin{array}{c}\text { mMRC } \\
\text { Retrospective data }\end{array}$ \\
\hline $\begin{array}{l}\text { STEWART [30], } \\
\text { UK }\end{array}$ & Cross-sectional & $\begin{array}{l}243 \text { patients with IPF } \\
\text { (140 patients without } \\
\text { disease progression } \\
\text { and } 103 \text { patients } \\
\text { with disease } \\
\text { progression) }\end{array}$ & $\begin{array}{l}\text { To determine if patient } \\
\text { response to a palliative } \\
\text { assessment survey could } \\
\text { predict disease progression } \\
\text { or death }\end{array}$ & $\begin{array}{c}\text { Physical: } \\
\text { ADL } \\
\text { Dyspnoea } \\
\text { Fatigue } \\
\text { GI symptoms (loss of } \\
\text { appetite) } \\
\text { Other (restless, } \\
\text { agitated) }\end{array}$ & SPARC EOL Questionnaire \\
\hline $\begin{array}{l}\text { TAKEYASU [31] } \\
\text { Japan }\end{array}$ & Retrospective & $\begin{array}{l}\text { Acute exacerbation } \\
\text { of end-stage } \\
\text { interstitial } \\
\text { pneumonia }(n=22)\end{array}$ & $\begin{array}{l}\text { To evaluate the efficacy and } \\
\text { safety of continuous } \\
\text { morphine infusion }\end{array}$ & $\begin{array}{l}\text { Physical: } \\
\text { Dyspnoea }\end{array}$ & $\begin{array}{l}\text { Subjective clinical } \\
\text { effectiveness rating }\end{array}$ \\
\hline
\end{tabular}




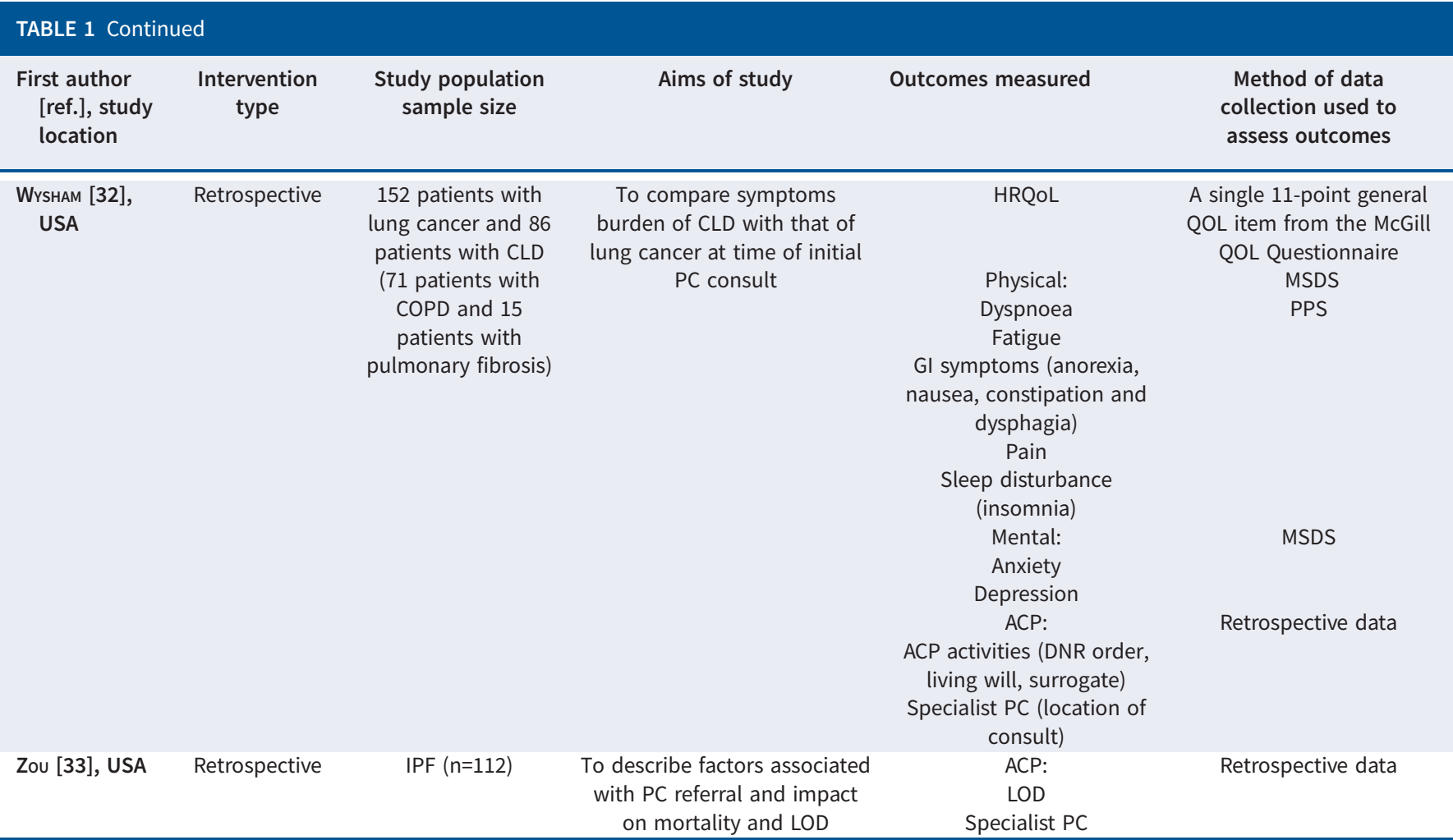

ILD: interstitial lung disease; GI: gastrointestinal; ACP: advance care planning; GOC: goals of care; LOD: location of death; PC: palliative care; SRPC: Swedish Register of Palliative Care; EOL: end of life; IPF: idiopathic pulmonary fibrosis; Wakefield-SADI: Wakefield Self-Assessment Depression Inventory; MDC: multidisciplinary care; mMRC: modified Medical Research Council Dyspnoea Scale; SDI: social deprivation index; PIF-ILD: progressive idiopathic fibrotic ILD; LOC: location of care; RCT: randomised controlled trial; HRQoL: health-related quality of life; QoL: quality of life; KB-ILD: Kings Brief ILD Questionnaire; SGRQ: St George's Respiratory Questionnaire; MRC: Medical Research Council Dyspnoea Scale; POS: Palliative Care Outcome Scale; HADS: Hospital Anxiety and Depression Scale; MDT: multidisciplinary team; CPR: cardiopulmonary resuscitation; ICU: intensive care unit; DNR: do not resuscitate; CHF: congestive heart failure; MND: motor neurone disease; CRQ: Chronic Respiratory Disease Questionnaire; LCADL: London Chest Activity of Daily Living; NRS: numeric rating scale; CSRI: Client Services Receipt Inventory; DNI: do not intubate; HFNC: high flow nasal cannula; NPPV: noninvasive positive pressure ventilation; STAS-J: Japanese version of the Support Team Assessment Schedule; mESAS: modified Edmonton symptom assessment; POS-S: Palliative Care Outcome Scale-Symptoms; ADL: activities of daily living; SPARC: modified Sheffield Profile for Assessment and Referral to Care; CLD: chronic lung disease; MSDS: McCorkle Symptom Distress Scale; PPS: palliative performance scale.

\section{Results}

\section{Literature review process}

We examined 4782 total articles (1667 from PubMed, 2874 from Embase and 241 from the Cochrane Library). Of these, 1294 duplicates were removed and there were 3488 remaining articles. 760 studies were deemed eligible for full-text review. Reviewers screened these full-text articles and selected 264 articles under the initial search strategy for palliative care outcomes in advanced lung disease. Reviewers (A.C. Moale, B. Seth and R.A. Gersten) identified those articles specific to ILD, which yielded 68 total articles. Of these, reviewers (A.C. Moale, B. Seth and R.A. Gersten) selected studies with quantitative results, for which there were initially a total of 21 . An updated search prior to paper submission yielded two additional articles, resulting in a total of 23 included articles (figure 2).

\section{Study designs of eligible studies}

Of the 23 eligible articles, the majority (16) were retrospective (70\%), followed by three randomised controlled trials (RCTs) or secondary analysis of an RCT (13\%), three cross-sectional studies or secondary analysis of a cross-sectional study (13\%) and one prospective study (4\%) (table 1).

\section{Outcomes assessed by domain}

Among the 23 eligible articles, six (26\%) assessed general QoL, 16 (70\%) assessed physical health, 11 (48\%) assessed mental health, six (26\%) assessed social health and 16 (70\%) evaluated ACP. The majority of articles (61\%) measured outcomes in more than one domain and $43 \%$ measured more than two domains. Among the 23 papers, 76 total analyses were performed (table 1). There were 25 distinct 
TABLE 2 Categorisation of domains and outcome measures

\begin{tabular}{|c|c|c|c|c|c|}
\hline Domains & HRQoL & Physical health & Mental health & Social health & ACP \\
\hline $\begin{array}{l}\text { Outcomes } \\
\text { measured }\end{array}$ & General HRQoL & $\begin{array}{c}\text { ADL } \\
\text { Dyspnoea } \\
\text { Energy } \\
\text { Fatigue } \\
\text { GI symptoms } \\
\text { Pain (including } \\
\text { intensity, } \\
\text { interference and } \\
\text { quality) } \\
\text { Physical function } \\
\text { Pruritus } \\
\text { Sexual function } \\
\text { Sleep } \\
\text { disturbance and } \\
\text { sleep-related } \\
\text { impairment } \\
\text { Other (including } \\
\text { cough, headache } \\
\text { and weight loss) }\end{array}$ & $\begin{array}{c}\text { Anxiety } \\
\text { Depression } \\
\text { Alcohol use } \\
\text { Anger } \\
\text { Cognitive function } \\
\text { (including. altered } \\
\text { mental status/delirium, } \\
\text { level of consciousness, } \\
\text { cognitive decline) } \\
\text { Life satisfaction } \\
\text { Meaning and purpose } \\
\text { Positive effect } \\
\text { Psychosocial illness } \\
\text { impact } \\
\text { Self-efficacy for } \\
\text { managing chronic } \\
\text { conditions } \\
\text { Smoking } \\
\text { Substance use }\end{array}$ & $\begin{array}{l}\text { Ability to participate in } \\
\text { social roles and activities } \\
\text { Companionship } \\
\text { Satisfaction with social } \\
\text { roles and activities } \\
\text { Social isolation } \\
\text { Social support }\end{array}$ & $\begin{array}{l}\text { ACP activities (code } \\
\text { status, advance } \\
\text { directives) } \\
\text { Expected versus } \\
\text { unexpected death } \\
\text { GOC discussions } \\
\text { (including prognosis } \\
\text { and end-of-life care) } \\
\text { LOC } \\
\text { LOD } \\
\text { Mechanical support } \\
\text { Specialist palliative } \\
\text { care }\end{array}$ \\
\hline $\begin{array}{l}\text { Instruments } \\
\text { used to } \\
\text { assess } \\
\text { domain }\end{array}$ & $\begin{array}{c}\text { KB-ILD } \\
\text { SGRQ } \\
\text { CRQ } \\
\text { EQ-5D } \\
\text { EQ-5D-HRQoL-VAS } \\
\text { POS } \\
\text { RAND-36 } \\
\text { A single 11-point general } \\
\text { QoL item from the McGill } \\
\text { QoL Questionnaire }\end{array}$ & $\begin{array}{c}\text { SRPC EOL } \\
\text { questionnaire } \\
\text { MRC } \\
\text { mMRC } \\
\text { D12 scale } \\
\text { POS } \\
\text { CRQ } \\
\text { LCADL } \\
\text { NRS } \\
\text { STAS-J } \\
\text { mESAS } \\
\text { RAND-36 } \\
\text { POS-S } \\
\text { SPARC } \\
\text { Subjective clinical } \\
\text { effectiveness } \\
\text { rating } \\
\text { MSDS } \\
\text { PPS }\end{array}$ & $\begin{array}{c}\text { SRPC EOL questionnaire } \\
\text { Wakefield-SADI } \\
\text { HADS } \\
\text { CRQ } \\
\text { mESAS } \\
\text { RAND-36 } \\
\text { NRS } \\
\text { MSDS }\end{array}$ & $\begin{array}{c}\text { SDI } \\
\text { CSRI } \\
\text { RAND-36 } \\
\text { LCADL }\end{array}$ & $\begin{array}{c}\text { SRPC EOL } \\
\text { Questionnaire }\end{array}$ \\
\hline Examples & $\begin{array}{l}\text { Self-reported overall QoL } \\
\text { in patients with chronic } \\
\text { respiratory disease } \\
\text { assessed via CRQ }\end{array}$ & $\begin{array}{l}\text { Symptoms of } \\
\text { dyspnoea } \\
\text { assessed via } \\
\text { mMRC }\end{array}$ & $\begin{array}{l}\text { Symptoms of anxiety/ } \\
\text { depression assessed via } \\
\text { HADS or Wakefield-SADI }\end{array}$ & $\begin{array}{c}\text { Retrospective review of } \\
\text { patient records regarding } \\
\text { ability of patient to } \\
\text { communicate with family/ } \\
\text { caregivers at EOL }\end{array}$ & $\begin{array}{l}\text { Retrospective review o } \\
\text { patient records } \\
\text { regarding presence of } \\
\text { DNR order prior to } \\
\text { patient death }\end{array}$ \\
\hline
\end{tabular}

HRQOL: health-related quality of life; ACP: advance care planning; ADL: activities of daily living; Gl: gastrointestinal; GOC: goals of care; LOC: location of care; LOD: location of death; KB-ILD: King's Brief ILD Questionnaire; SGRQ: St George's Respiratory Questionnaire; CRQ: Chronic Respiratory Disease Questionnaire; VAS: visual analogue scale; POS: Palliative Care Outcome Scale; QoL: quality of life; SRPC: Swedish Register of Palliative Care; EOL: end of life; MRC: Medical Research Council Dyspnoea Scale; mMRC: modified MRC; LCADL: London Chest Activity of Daily Living; NRS: Numerical Rating Scale; STAS-J: Japanese version of the Support Team Assessment Schedule; mESAS: modified Edmonton symptom assessment; POS-S: Palliative Care Outcome Scale-Symptoms; SPARC: modified Sheffield Profile for Assessment and Referral to Care Questionnaire; MSDS: McCorkle Symptom Distress Scale; PPS: palliative performance scale; Wakefield-SADI: Wakefield Self-Assessment Depression Inventory; HADS: Hospital Anxiety and Depression Scale; SDI: social deprivation index; CSRI: Client Services Receipt Inventory; DNR: do not resuscitate.

instruments used in 53 analyses (70\%). Retrospective data, as defined above, was used in 23 analyses (30\%). Instruments may be used to assess multiple domains. Table 3 describes each instrument and identifies the domains authors used the instrument to assess.

\section{HRQOL}

General HRQoL was assessed in six articles (26\%) with eight distinct instruments (table 1). Of these eight instruments, only King's Brief Interstitial Lung Disease (KB-ILD) and St George’s Respiratory Disease Questionnaire (SGRQ) are validated in the ILD population (table 3) [16, 38, 49]. The Chronic Respiratory 


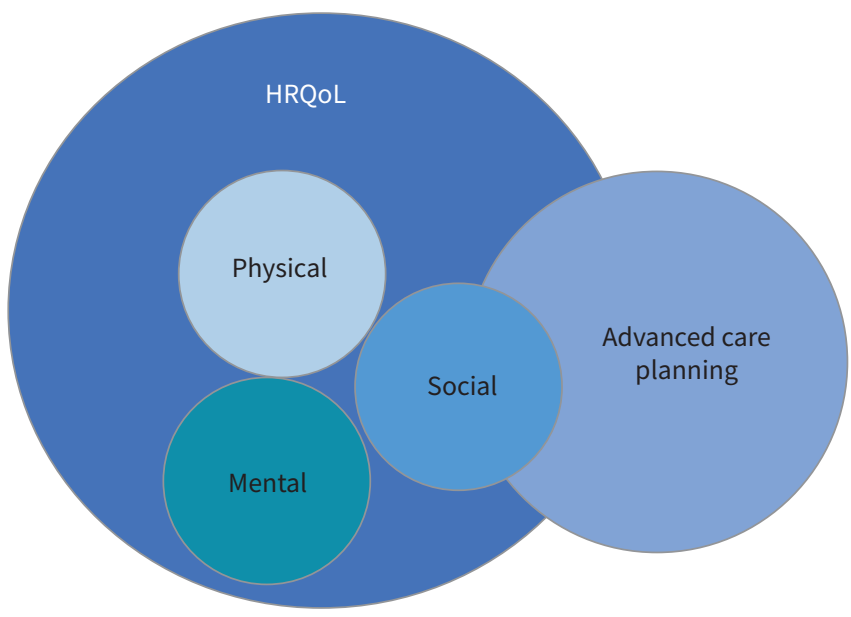

FIGURE 1 Physical, mental and social domains as categorised by patient-reported outcomes measurement information system (PROMIS) adult self-reported health. General health-related quality of life (HRQOL) was added as a fourth domain, realising that although HRQoL includes physical, mental and social functioning, it can also be assessed generally. Advance care planning was added as a fifth domain.

Disease Questionnaire and the RAND 36-Item Health Survey (RAND-36) were used in multiple studies. The remaining six instruments (table 2) were each used only once. Retrospective data was never used to assess HRQoL.

\section{Physical health}

Physical health was assessed in 16 articles (70\%) with 16 distinct instruments. The modified Medical Research Council Dyspnoea Scale was used four times and the Numerical Rating Scale was used three times to measure physical health. The London Chest Activity of Daily Living, the modified Edmonton Symptom Assessment System (mESAS), RAND-36 and the Palliative Care Outcome Scale were each used twice. The remaining 10 instruments (table 2) were all used once. Retrospective data was used four times.

Among the physical health outcomes, dyspnoea was the most commonly measured (15 times). Other symptoms (including restlessness, agitation, cough and dry mouth) were assessed nine times. Fatigue and pain were assessed eight times. Gastrointestinal symptoms (including anorexia, nausea, diarrhoea, constipation, dysphagia and loss of appetite) were assessed seven times. Sleep disturbances, including insomnia, were assessed six times. Physical function and activities of daily living were assessed a total of four times. Energy level was only assessed once.

\section{Mental health}

Mental health was assessed in 11 articles (48\%) with eight distinct instruments. The Hospital Anxiety and Depression Scale was used three times and the mESAS and RAND-36 were each used twice to measure mental health. The remaining five instruments (table 2) were each used once. While some of these instruments have been validated in the general or cancer population, none of these instruments are validated in the ILD population. Retrospective data was used three times. Anxiety and depression were each assessed nine times. Cognitive function (including confusion, loss of consciousness and delirium) was assessed three times.

\section{Social health}

Social health was assessed in six articles (26\%) with four distinct instruments. RAND-36 was used twice. The remaining three instruments (table 2) were each used once. Retrospective data was used once. Ability to participate in social roles and activities was assessed three times. Social isolation, social function and other (including receipt of health, voluntary or social care services) were each assessed once.

$A C P$

ACP was assessed in 16 articles (70\%). The Swedish Register of Palliative Care end-of-life questionnaire was the only instrument used. Retrospective data was used 15 times. Specialist palliative care was assessed 11 times, location of death was assessed nine times and ACP activities (including code status and advance directive discussions) were assessed eight times. Mechanical support (including intubation and removal of 
TABLE 3 Palliative care outcome measures in ILD

\begin{tabular}{|c|c|}
\hline Name of instrument & Description \\
\hline $\begin{array}{l}\text { SRPC EOL Questionnaire } \\
\text { [5] }\end{array}$ & $\begin{array}{l}\text { National quality register of the care } \\
\text { patients with ILD collected through a } \\
\text { EOL questionnaire completed } \\
\text { retrospectively by a care provider } \\
\text { within a week of a patient's death }\end{array}$ \\
\hline Wakefield-SADI [13] & Screening tool for major depression \\
\hline MRC $[16,35]$ & $\begin{array}{l}\text { Dyspnoea scale used to quantify } \\
\text { disability associated with } \\
\text { breathlessness, with higher scores } \\
\text { indicating greater impairment }\end{array}$ \\
\hline mMRC [35] & $\begin{array}{c}\text { Similar to original MRC but with } \\
\text { breathlessness scales ranging from } \\
\text { grade } 0 \text { to } 4\end{array}$ \\
\hline SDI [36] & $\begin{array}{l}\text { Factor score based on Canadian } \\
\text { census data to reflect the deprivation } \\
\text { of relationships among individuals in } \\
\text { the family, the workplace and the } \\
\text { community }\end{array}$ \\
\hline KB-ILD [37] & $\begin{array}{c}\text { Self-reported, ILD-specific, HRQoL } \\
\text { questionnaire }\end{array}$ \\
\hline SGRQ $[16,38,39]$ & $\begin{array}{c}\text { Measures impact on overall health, } \\
\text { daily life, and perceived well-being, } \\
\text { with higher scores indicating greater } \\
\text { disability }\end{array}$ \\
\hline
\end{tabular}

$\begin{array}{lc}\text { D12 Scale [39] } & \begin{array}{c}\text { Provides overall score for } \\ \text { breathlessness severity }\end{array} \\ \text { POS [40] } & \begin{array}{l}\text { Group of tools used to measure PC } \\ \text { needs of patients and their families }\end{array}\end{array}$

POS-S $[40,41]$

HADS [42]

CRQ [19]

EQ-5D-HRQOL-VAS $[19,43]$
EQ-5D questionnaire as above, plus a VAS that allows respondents to score a characteristic or attitude across a continuum of values
POS version specific to measuring a number of different symptoms

Assess for depression and anxiety

12 physical and affective items on a scale of none (0), mild

(1), moderate (2), severe (3) 10 items on physical symptoms, emotional, psychological and spiritual needs, and provision of information and support

10 symptoms and two open questions about the symptom that affected the patient the most and that has improved the most

14 items on depression and Mental health anxiety

20 items on dyspnoea, fatigue, emotional function and mastery questionnaire measuring physical and emotional aspects, with higher scores indicating better HRQoL Self-reported questionnaire used to characterise current health states

15 items on mobility, self-care, usual activities, pain/ discomfort and anxiety/ depression

15 items as above and VAS

health health HRQoL

\section{Domains \\ Population in which}

assessed validated

with

instrument

Physical

Mental

ACP

N/A

Mental health General populations

Physical Respiratory diseases, health particularly COPD

Physical Respiratory diseases, health particularly COPD

Social health

N/A

HRQoL

ILD

HRQoL Designed for obstructive airway disease

Also found to be valid in restrictive lung disease with modified version SGRQ specific for IPF (SGRQ-I) validated in IPF

Physical N/A

HRQoL Wide spectrum of cancer Physical and non-cancer diseases

Physical Wide spectrum of cancer health and non-cancer diseases Physical including mild-to-moderate health non-cystic fibrosis, Mental health bronchiectasis and COPD HRQoL N/A; generic measure of health conditions N/A 
TABLE 3 Continued

Name of instrument

Description

Number of items

Domains

assessed

with

instrument

\begin{tabular}{c}
\hline Assesses dyspnoea-related \\
impairment in ADL
\end{tabular}

0 to 10 NRS A scale used to quantify an attribute
CSRI [19] Collects information on range of
services

\section{Japanese version of Support Team Assessment Schedule (STAS-J) $[21,44]$}

RAND-36 [45] General HRQoL survey outcomes of PC
Adapted from STAS, which assesses
Physical

15 items on self-care, and domestic, physical and social activities

0 to 10 scale

health

Physical Social health health Mental health

Variable items on health, voluntary and social care services

9 core items or up to $20 \quad$ Physical General populations optional items on physical, psychosocial, spiritual, communication, planning, family concerns and service aspects

36 items on physical functioning, role limitations cause by physical health problems, role limitations caused by emotional problems, social functioning, emotional well-being energy/

fatigue, pain and general health perceptions

\begin{tabular}{cc}
\hline mESAS [25] & $\begin{array}{c}\text { Self-rated, numerical-rating, } \\
\text { symptom-based scale developed to } \\
\text { assess symptoms }\end{array}$ \\
SPARC Questionnaire & $\begin{array}{c}\text { Screening tool which provides a } \\
\text { profile of needs to identify patients } \\
\text { w46] }\end{array}$ \\
$\begin{array}{c}\text { Subjective clinical } \\
\text { effectiveness rating }\end{array}$ & $\begin{array}{c}\text { Subjective rating of good, moderate, } \\
\text { poor, or unknown to measure } \\
\text { [31] }\end{array}$ \\
MQoL & symptom relief \\
Evaluates QoL
\end{tabular}

12 items on symptoms, one of general well-being, and standardised body diagram where areas of pain can be marked

45 items on physical, psychological, social and spiritual needs 1 item

Social health

COPD may also be valid in ILD and cancer

N/A N/A health

\section{HRQoL \\ Physical \\ health}

Mental health

Social health
Population in which validated 


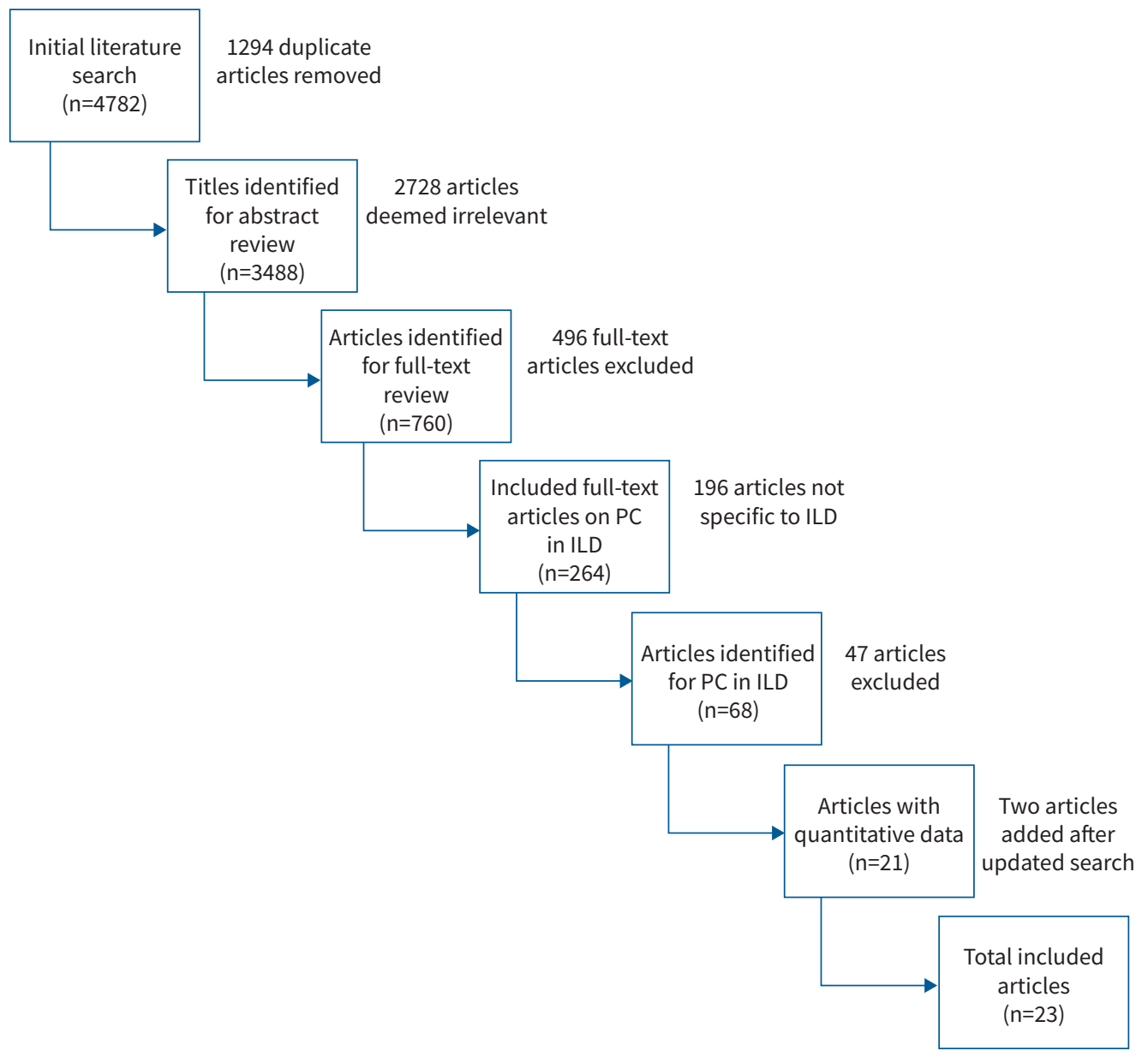

FIGURE 2 Literature flow of articles in the scoping review. PC: palliative care: ILD: interstitial lung disease.

endotracheal tube) was assessed four times. Less commonly, goals of care discussions (including prognosis and end-of-life care) and location of care were assessed three times. Expected versus unexpected death was only assessed once.

\section{Discussion}

This scoping review delineates multi-dimensional outcome measures of palliative care in patients with ILD. Palliative care is a comprehensive approach to improving the QoL of patients with serious illness through the identification and assessment of physical, psychosocial and spiritual issues, which may evolve throughout the course of an illness [9]. Palliative care has shown to benefit a number of chronic medical conditions and has been integrated into standard care. In general, for adults with advanced illness, palliative care may improve a patient's QoL, symptom burden, patient satisfaction with care and chances of dying in a preferred location [50]. For patients with congestive heart failure (CHF), palliative care has demonstrated statistically and clinically significant improvements in QoL and symptom burden leading to the incorporation of palliative care into the treatment guidelines for CHF [51, 52]. Palliative care in lung cancer improves QoL, lessens depressive symptoms and actually increases median survival despite less aggressive care at the end of life [53]. When palliative care is embedded in an ILD clinic, there are significantly higher rates of ACP. Higher rates of ACP increase the likelihood of patients receiving end-of-life care in concordance with their preferences and may increase satisfaction with healthcare and decrease healthcare cost [54]. The importance of palliative care for patients with ILD is of increasing interest [9]. While we speculate that palliative care improves QoL in patients with ILD, we currently lack conclusive data. Evaluation of outcome measurement plays a significant role in assessing quality palliative care interventions. Currently, the diversity of outcome measures is vast. This lack of uniformity makes the selection of appropriate outcome measures and interpretation of results across studies challenging. 
The majority of articles included were retrospective analyses. Only three RCTs have been conducted on this important subject. RCTs are the "gold standard" for study design [55], and we hope to see the emergence of high-quality trials to further evaluate the impact of palliative care interventions in ILD. Standardisation of domains and outcome measures is a key first step to expanding this evidence base.

In this study, we organised data into the PROMIS domains of physical health, mental health and social health. Not all critically important data to the field of palliative care fit into the PROMIS domains, thus we proposed two new domains of general HRQoL and ACP (figure 1 and table 2). Physical health was the most frequently assessed domain, with dyspnoea being most often assessed (table 3). This may be because physical symptoms are more easily and commonly described by patients, and clinicians often focus more on patients' symptom burden and physical limitations than psychologic well-being [56]. Physical health was primarily evaluated via instruments, which is likely due to the greater number of standardised instruments designed to assess physical health than any other QoL domain.

ACP was also frequently assessed. However, the majority of assessments were conducted by retrospective chart review rather than by use of a specific instrument. In order to consistently assess patients' goals, which govern care and treatment options, it is critical to develop standardised, validated instruments to assess adequate ACP rather than relying on inconsistent provider-specific documentation. No such tool currently exists.

After physical health and ACP, mental health was most frequently evaluated. Many patients with chronic diseases, such as ILD, experience mental distress; however, symptom assessment can be challenging [56]. Anxiety and depression were commonly assessed. Social health was the least frequently evaluated domain. Patients’ psychosocial issues may emotionally burden clinicians [56] and are often overlooked, despite a recent systematic review demonstrating the critical role of social health from patients' perspectives [57].

Despite the variety of existing outcomes measures, most studies used generic outcome measures not specifically designed for or validated in ILD to evaluate multiple domains. On the contrary, there are widely used and validated outcome measures that are not being consistently used in palliative care research in patients with ILD. Of the two instruments validated in ILD (KB-ILD and SGRQ), both assess the general HRQoL domain. Some articles introduced new unvalidated instruments. Ideally, validated instruments specific to the domain(s) under investigation should be selected. For instruments that measure several domains, clear delineation of section scores by domain would allow for independent assessment of more than one domain per instrument. Likewise, established instruments are preferred to the development of new instruments [10] in order to minimise collection burden [58]. It may be adventitious to validate established outcome measures in the ILD population.

Currently, there are no standardised outcome measures to assess palliative care interventions. In order to conduct urgently needed research on the impact of palliative care interventions in ILD, we must establish a core set of outcome measures. This would include consensus agreement upon our five proposed domains, validation of more instruments in the ILD population and concordance on which domains may be assessed with each instrument. We hope this scoping review serves as a first step in accomplishing these goals.

There are strengths and limitations to this scoping review. To our knowledge, this is the only review of palliative care outcome measures in ILD patients. Our initial search was broad, including all studies evaluating palliative care interventions in advanced lung disease. We subsequently selected studies specific to the ILD population that included quantitative data. Although every effort was made to include all eligible studies, it remains possible that eligible studies may have been unintentionally omitted. For example, although palliative care encompasses end-of-life care and symptom management, we did not specifically search for "end-of-life care" or particular interventions focused on symptom control. Additionally, since this study only evaluated quantitative data, valuable qualitative data that may be helpful in understanding patient-centred outcome measures were not included. Furthermore, although the caregiver burden is high and the development of caregiver outcome measures is important, this review focused on patient outcome measures. It should also be acknowledged that only articles available in English were included, which primarily biases towards studies conducted in the UK and the USA. Palliative care is dedicated to the provision of culturally sensitive care and we may have omitted studies from other countries that might explore interventions or outcomes of particular importance in other cultures.

\section{Conclusion}

This scoping review of 23 articles demonstrates the current diversity of palliative care outcome measures in ILD. We identified 25 distinct instruments and categorised them under one or more of five domains. We 
introduce a new domain of ACP given the critical need for standardised assessment of patients' goals and preferences. The majority of these instruments are generic outcome measures, assessing more than one domain. This review can serve as a reference for researchers seeking guidance in choosing outcome measures of palliative care interventions in the ILD population. It is important to recognise that the heterogeneity of outcome measures creates challenges in selecting appropriate outcome measures and comparing results across studies. Development and utilisation of a core set of outcome measures is urgently needed to advance research aimed at improving patient-centred outcomes and quality of care for patients with ILD.

Provenance: Submitted article, peer reviewed

Acknowledgements: We thank clinical informationist Carrie Price, MLS (Johns Hopkins University, Baltimore, MD, USA) for her assistance with our literature search.

Conflict of interest: None declared.

\section{References}

1 Duchemann B, Annesi-Maesano I, de Naurois C J, et al. Prevalence and incidence of interstitial lung diseases in a multi-ethnic county of Greater Paris. Eur Respir J 2017; 50: 1602419.

2 Raghu G, Collard HR, Egan JJ, et al. An official ATS/ERS/JRS/ALAT Statement: idiopathic pulmonary fibrosis: evidence-based guidelines for diagnosis and management. Am J Respir Crit Care Med 2011; 183: 788-824.

3 Ojanguren I, Morell F, Ramón MA, et al. Long-term outcomes in chronic hypersensitivity pneumonitis. Allergy 2019; 74: 944-952.

4 Hyldgaard C, Ellingsen T, Hilberg $\mathrm{O}$, et al. Rheumatoid arthritis-associated interstitial lung disease: clinical characteristics and predictors of mortality. Respiration 2019; 98: 455-460.

5 Ahmadi Z, Wysham NG, Lundström S, et al. End-of-life care in oxygen-dependent ILD compared with lung cancer: a national population-based study. Thorax 2016; 71: 510-516.

6 Kreuter M, Swigris J, Pittrow D, et al. The clinical course of idiopathic pulmonary fibrosis and its association to quality of life over time: longitudinal data from the INSIGHTS-IPF registry. Respir Res 2019; 20: 59.

7 Matsunuma $\mathrm{R}$, Takato $\mathrm{H}$, Takeda $\mathrm{Y}$, et al. Patients with end-stage interstitial lung disease may have more problems with dyspnea than end-stage lung cancer patients. Indian J Palliat Care 2016; 22: 282-287.

8 Kreuter M, Bendstrup E, Russell AM, et al. Palliative care in interstitial lung disease: living well. Lancet Respir Med 2017; 5: 968-980.

9 Lanken PN, Terry PB, DeLisser HM, et al. An official American Thoracic Society clinical policy statement: palliative care for patients with respiratory diseases and critical illnesses. Am J Respir Crit Care Med 2008; 177: 912-927.

10 Bausewein C, Daveson B, Benalia H, et al. Outcome Measurement in Palliative Care. The Essentials. http:// mailsystem.palliatief.be/accounts/143/attachments/Nieuwsflash/25_prisma_essentials.pdf Date last accessed: 14 March 2021.

11 Reeve BB, Hays RD, Bjorner JB, et al. Psychometric evaluation and calibration of health-related quality of life item banks: plans for the Patient-Reported Outcomes Measurement Information System (PROMIS). Med Care 2007; 45: S22-S31.

12 Rothrock NE, Hays RD, Spritzer K, et al. Relative to the general US population, chronic diseases are associated with poorer health-related quality of life as measured by the Patient-Reported Outcomes Measurement Information System (PROMIS). J Clin Epidemiol 2010; 63: 1195-1204.

13 Akhtar AA, Ali MA, Smith RP. Depression in patients with idiopathic pulmonary fibrosis. Chron Respir Dis 2013; 10: 127-133.

14 Archibald N, Bakal JA, Richman-Eisenstat J, et al. Early integrated palliative care bundle impacts location of death in interstitial lung disease: a pilot retrospective study. Am J Hosp Palliat Med 2021; 38: 104-113.

15 Bajwah S, Higginson IJ, Ross JR, et al. Specialist palliative care is more than drugs: a retrospective study of ILD patients. Lung 2012; 190: 215-220.

16 Bajwah S, Ross JR, Wells AU, et al. Palliative care for patients with advanced fibrotic lung disease: a randomised controlled phase II and feasibility trial of a community case conference intervention. Thorax 2015; 70: 830-839.

17 Barratt SL, Morales M, Speirs T, et al. Specialist palliative care, psychology, interstitial lung disease (ILD) multidisciplinary team meeting: a novel model to address palliative care needs. BMJ Open Respir Res 2018; 5: e000360.

18 Brown CE, Engelberg RA, Nielsen EL, et al. Palliative care for patients dying in the intensive care unit with chronic lung disease compared with metastatic cancer. Ann Am Thorac Soc 2016; 13: 684-689.

19 Higginson IJ, Bausewein C, Reilly CC, et al. An integrated palliative and respiratory care service for patients with advanced disease and refractory breathlessness: a randomised controlled trial. Lancet Respir Med 2014; 2: 979-987. 
20 Kalluri M, Claveria F, Ainsley E, et al. Beyond idiopathic pulmonary fibrosis diagnosis: multidisciplinary care with an early integrated palliative approach is associated with a decrease in acute care utilization and hospital deaths. J Pain Symptom Manage 2018; 55: 420-426.

21 Koyauchi T, Hasegawa H, Kanata K, et al. Efficacy and tolerability of high-flow nasal cannula oxygen therapy for hypoxemic respiratory failure in patients with interstitial lung disease with do-not-intubate orders: a retrospective single-center study. Respiration 2018; 96: 323-329.

22 Liang Z, Hoffman LA, Nouraie M, et al. Referral to palliative care infrequent in patients with idiopathic pulmonary fibrosis admitted to an intensive care unit. J Palliat Med 2017; 20: 134-140.

23 Lindell KO, Liang Z, Hoffman LA, et al. Palliative care and location of death in decedents with idiopathic pulmonary fibrosis. Chest 2015; 147: 423-429.

24 Rajala K, Lehto JT, Saarinen M, et al. End-of-life care of patients with idiopathic pulmonary fibrosis. $B M C$ Palliat Care 2016; 15: 788.

25 Rajala K, Lehto JT, Sutinen E, et al. mMRC dyspnoea scale indicates impaired quality of life and increased pain in patients with idiopathic pulmonary fibrosis. ERJ Open Res 2017; 3: 00084-2017.

26 Rajala K, Lehto JT, Sutinen E, et al. Marked deterioration in the quality of life of patients with idiopathic pulmonary fibrosis during the last two years of life. BMC Pulmon Med 2018; 18: 788.

27 Reilly CC, Bausewein C, Garrod R, et al. Breathlessness during daily activity: the psychometric properties of the London Chest Activity of Daily Living Scale in patients with advanced disease and refractory breathlessness. Palliat Med 2017; 31: 868-875.

28 Rush B, Berger L, Celi LA. Access to palliative care for patients undergoing mechanical ventilation with idiopathic pulmonary fibrosis in the United States. Am J Hosp Palliat Med 2018; 35: 492-496.

29 Smallwood N, Mann J, Guo H, et al. Patients with fibrotic interstitial lung disease receive supportive and palliative care just prior to death. Am J Hosp Palliat Med 2021; 38: 154-160.

30 Stewart I, McKeever T, Braybrooke R, et al. Patient reported distress can aid clinical decision making in idiopathic pulmonary fibrosis: analysis of the PROFILE cohort. Eur Respir J 2019; 53: 1801925.

31 Takeyasu M, Miyamoto A, Kato D, et al. Continuous Intravenous Morphine Infusion for Severe Dyspnea in Terminally III Interstitial Pneumonia Patients. Am J Respir Crit Care Med 2015; 191:A3324.

32 Wysham NG, Cox CE, Wolf SP, et al. Symptom burden of chronic lung disease compared with lung cancer at time of referral for palliative care consultation. Ann Am Thorac Soc 2015; 12: 1294-1301.

33 Zou RH, Nouraie M, Chen X, et al. Assessing patterns of palliative care referral and location of death in patients with idiopathic pulmonary fibrosis: a sixteen-year single-center retrospective cohort study. J Palliat Med 2019; 22: 538-544.

34 Hahn EA, DeVellis RF, Bode RK, et al. Measuring social health in the patient- reported outcomes measurement information system (PROMIS): item bank development and testing. Qual Life Res 2010; 19: 1035-1044.

35 Williams N. The MRC breathlessness scale. Occup Med 2017; 67: 496-497.

36 Fransoo R, Mahar A, Anderson A, et al. The 2019 RHA Indicators Atlas. Winnipeg, Manitoba Centre for Health Policy, 2019.

37 Nolan CM, Birring SS, Maddocks M, et al. Kings brief interstitial lung disease questionnaire: responsiveness and minimum clinically important difference. Eur Respir J 2019; 54: 1600281.

38 American Thoracic Society. St. George's Respiratory Questionnaire (SGRQ). www.thoracic.org/members/ assemblies/assemblies/srn/questionaires/sgrq.php Date last accessed: 14 March 2021.

39 Yorke J, Moosavi SH, Shuldham C, et al. Quantification of dyspnoea using descriptors: development and initial testing of the Dyspnoea-12. Thorax 2010; 65: 21-26.

40 Palliative care Outcome Scale. The Palliative care Outcome Scale and outline for new and current users. https://pos-pal.org/maix/pos-and-ipos-summary.php Date last accessed: 14 March 2021.

41 Azhar A, Bruera E. Outcome measurement and complex physical, psychosocial and spiritual experiences of death and dying. Ann Palliat Med 2018;7:S231-S243.

42 Vodanovich DA, Bicknell TJ, Holland AE, et al. Validity and reliability of the chronic respiratory disease questionnaire in elderly individuals with mild to moderate non-cystic fibrosis bronchiectasis. Respiration 2015; 90: 89-96.

43 American Thoracic Society. EuroQol (EQ-5D). https://qol.thoracic.org/sections/instruments/ae/pages/euroqol. html Date last updated: August 1990; date last accessed: 14 March 2021.

44 Bausewein C, Le Grice C, Simon S, et al. The use of two common palliative outcome measures in clinical care and research: a systematic review of POS and STAS. Palliat Med 2011; 25: 304-313.

45 Hays RD, Morales LS. The RAND-36 measure of health-related quality of life. Ann Med 2001; 33: 350-357.

46 Ahmed N, Hughes P, Winslow M, et al. Feasibility study of the sheffield profile for assessment and referral for care. BMJ Supportive \& Palliative Care 2012; 2: Suppl. 1, A43-A44.

47 American Thoracic Society. Symptom Distress Scale. https:/qol.thoracic.org/sections/instruments/pt/pages/ sds.html Date last updated: May 1999; date last accessed: 14 March 2021.

48 Lau F, Downing M, Lesperance M, et al. Using the palliative performance scale to provide meaningful survival estimates. J Pain Symptom Manage 2009; 38: 134-144. 
49 Prior TS, Hoyer N, Shaker SB, et al. Validation of the IPF-specific version of St George's Respiratory Questionnaire. Respir Res 2019; 20: 199.

50 Bajwah S, Oluyase AO, Yi D, et al. The effectiveness and cost-effectiveness of hospital-based specialist palliative care for adults with advanced illness and their caregivers. Cochrane Database Syst Rev2020; 9: CD012780.

51 Yancy CW, Jessup M, Bozkurt B, et al. 2017 ACC/AHA/HFSA Focused Update of the 2013 ACCF/AHA Guideline for the Management of Heart Failure: a Report of the American College of Cardiology/American Heart Association Task Force on Clinical Practice Guidelines and the Heart Failure Society of America. J Am Coll Cardiol 2017; 70: 776-803.

52 Kavalieratos D, Corbelli J, Zhang DI, et al. Association between palliative care and patient and caregiver outcomes: a systematic review and meta-analysis. JAMA 2016; 316: 2104-2114.

53 Temel JS, Greer JA, Muzikansky A, et al. Early palliative care for patients with metastatic non-small-cell lung cancer. New Eng J Med 2010; 363: 733-742.

54 Houben CHM, Spruit MA, Groenen MTJ, et al. Efficacy of advance care planning: a systematic review and meta-analysis. J Am Med Dir Assoc 2014; 15: 477-489.

55 Williamson PR, Altman DG, Bagley H, et al. The COMET Handbook: version 1.0. Trials 2017; 18: 280.

56 Turner J, Kelly B. Emotional dimensions of chronic disease. West J Med 2000; 172: 124-128.

57 McCaffrey N, Bradley S, Ratcliffe J, et al. What aspects of quality of life are important from palliative care patients' perspectives? A systematic review of qualitative research. J Pain Symptom Manage 2016; 52: 318-328.

58 de Wolf-Linder S, Dawkins M, Wicks F, et al. Which outcome domains are important in palliative care and when? An international expert consensus workshop, using the nominal group technique. Palliat Med 2019; 33: $1058-1068$. 